\title{
Polarization of diffracted optical wave in the case of acousto-optic diffraction on the longitudinal acoustic wave in fused silica
}

\author{
Krupych O., Martynyuk-Lototska I., Orykhivskyi I., Kostyrko M. and Vlokh R. \\ O. G. Vlokh Institute of Physical Optics, 23 Dragomanov Street, 79005 Lviv, \\ Ukrainevlokh@ifo.lviv.ua
}

Received: 16.02 .2021

\begin{abstract}
We report on polarimetric studies for the diffracted light originated from acousto-optic (AO) diffraction at a longitudinal acoustic wave. A particular case of intermediate Bragg-Raman-Nath diffraction regime in fused silica is considered. We find that for the incident circularly polarized optical waves the ellipticity of the diffracted wave is determined by the ratio of elasto-optic coefficients $p_{11}$ and $p_{12}$, while for the incident linearly polarized waves this ratio is determined by the azimuth of diffracted wave. The ratio of coefficients determined in this way agrees well with the literature data. We detect a small deviation from linear polarization for the diffracted optical waves under condition when the incident waves are linearly polarized. This deviation can be caused by changing phase difference of the diffracted eigenmodes.
\end{abstract}

Keywords: acousto-optic diffraction, light polarization, fused silica

UDC: $535.42+535.513+534.23$

\section{Introduction}

Acousto-optic (AO) diffraction is a well-known phenomenon that consists in diffraction of optical waves on the phase grating caused by an acoustic wave (AW) via elasto-optic (EO) effect (see, e.g., Refs. [1-3]). The characteristics of diffracted wave depend on many parameters. So, the efficiency of AO diffraction is given by the formula

$$
\eta=\sin ^{2}\left(\frac{\pi}{\lambda \cos \theta_{B}} \sqrt{\frac{n^{6} p_{e f}^{2} P_{a}}{\rho v^{3}} \frac{l}{2 b}}\right)=\sin ^{2}\left(\frac{\pi}{\lambda \cos \theta_{B}} \sqrt{M_{2} P_{a} \frac{l}{2 b}}\right),
$$

where

$$
M_{2}=\frac{n^{6} p_{e f f}^{2}}{\rho v^{3}}
$$

is the AO figure of merit, $\rho$ the material density, $p_{\text {eff }}$ the effective EO coefficient, $\lambda$ the wavelength of optical radiation, $\theta_{B}$ the Bragg angle, $v$ and $P_{a}$ denote respectively the velocity and the power of the AW, and $l$ and $b$ the interaction length and the height of acoustic beam, respectively. In the case of $\eta \ll 1$, Eq. (1) can be simplifies to

$$
\eta=M_{2} \frac{\pi^{2} l}{2 \lambda^{2} b \cos ^{2} \theta_{B}} P_{a} .
$$

Eq. (3) demonstrates that the efficiency of AO diffraction depends on the AO figure of merit and a number of geometric parameters. In its turn, the AO figure of merit given by Eq. (2) depends on the constitutive parameters of a medium such as refractive indices, EO coefficients, material density and elastic-stiffness coefficients determining the velocity of AWs. Then the intensity $I$ of 
the diffracted optical wave is determined by the efficiency of AO diffraction as $I=\eta I_{0}$, where $I_{0}$ is the incident light intensity.

The diffracted optical wave is also characterized by its polarization and phase. The polarization of the diffracted wave depends on the type of diffraction. When the optical eigenwaves are linearly polarized, the diffracted-wave polarization can be parallel (in case of isotropic type of diffraction) or perpendicular (in case of anisotropic diffraction) to the polarization of incident optical wave, while the latter polarization is parallel to one of the eigenvectors of optical-impermeability tensor $B_{i j}$. Let us consider the isotropic diffraction type. In general, the diffracted wave has to be elliptically polarized when the incident optical wave is linearly polarized at some nonzero angle with respect to the eigenvectors of the impermeability tensor. Then the elliptical polarization is in fact caused by different EO coefficients (or refractive indices) that correspond to different orthogonal eigenvectors of the $B_{i j}$ cross-section, which is perpendicular to the diffracted wave vector. This follows from the relation written for the electric field of the diffracted wave:

$$
E_{i}=B_{i j} D_{j}=p_{i j k l} e_{k l}^{\Omega},
$$

where $D_{j}$ is the electrical induction of the incident optical wave, ${ }_{i j k l}$ the EO coefficients, $e_{k l}^{\Omega}$ the strain caused by the AW with the frequency $\Omega$. The ellipticity of the diffracted optical wave is defined solely by the ratio of EO coefficients only in the case when the phase difference between the diffracted eigenwaves with mutually perpendicular polarizations is not changed. Of course, the phase of the diffracted wave is changed due to Doppler effect $\left(\omega_{i} \pm \Omega\right)$ whenever the diffraction occurs on running AW. This additional phase shift is equal to $\delta= \pm \Omega t$, where ${ }^{\omega_{i}}$ denotes the frequency of the incident optical wave and ${ }^{t}$ the time. However, this shift remains the same for the both diffracted waves with orthogonal polarizations. As a consequence, it makes no impact on the polarization of diffracted waves.

It is worthwhile that possible changes in the phases of diffracted optical waves, which can occur in the course of AO diffraction, have been rarely examined by researchers. One of examples is the work [4] where it has been shown that, at the Bragg AO diffraction, the domains with higher spatial coherence diffract with substantially higher efficiency than the domains with lower spatial coherence. It is also known [5] that, in the course of the Bragg interaction, the degrees of spatial coherence of the diffracted light beams can be controlled by either intensity or frequency of the AW. Moreover, it has been demonstrated that the zero-order wave is less coherent than the incident optical wave, whereas the diffracted wave of the order -1 is more coherent than the incident light. Here the incident wave is partially coherent spatially. At the same time, the degree of coherence of the diffracted optical wave is the same as that of the incident wave, whenever the incident wave is highly coherent. Moreover, when the incident wave is highly incoherent, the degree of coherence of the zero-order diffracted wave is the same as that of the incident optical wave, whereas the wave of the order -1 becomes more coherent than the incident one.

All of these results imply that the phase of the diffracted optical wave undergoes some change in the course of AO diffraction or, at least, the degree of coherence for the diffracted wave can increase. The authors [6] have found that the optical phase of each order of the Raman-Nath diffracted light fluctuate with the AW. As a result, the wavefront of each order of the diffracted 
light is modulated. It has been shown in our recent work [7] that the phase structure of the optical vortex is preserved at the AO diffraction. However, this conclusion has been confirmed only qualitatively, following from observations of spiral-like interference patterns which occur under interference of the diffracted vortex beam with the reference Gaussian beam. Notice that the change in the phase of the diffracted beam cannot be revealed when the phase depends nonlinearly on the tracing angle, which changes by $2 \pi$ around the vortex core. All of the facts discussed above indicate that the problem of optical phase changes in the course of AO diffraction has not yet been studied in sufficient detail.

The present work is devoted to the polarization of AO-diffracted wave in the simplest case of AO diffraction at the longitudinal AW in isotropic media.

\section{Experimental procedures and results}

We studied the behaviour of light polarization using the AO diffraction of polarized light in fused silica. A longitudinal AW propagated along 'horizontal' direction, which coincided with the $\mathrm{X}$ axis of laboratory coordinate system. Lithium niobate transducer with the dimensions $5 \times 3 \mathrm{~mm} 2$ and the resonant frequency $50 \mathrm{MHz}$ was used to excite the AW. The incident light was directed normally to the AW in the horizontal plane, i.e. along the $\mathrm{Z}$ axis. The AO diffraction was studied in the intermediate (Bragg-Raman-Nath) regime. The Klein-Cook parameter Q was close to the unity $(\mathrm{Q}=0.98)$. The probing light had both linear and circular polarizations. Polarization states of the diffracted beams of the orders $0,+1$ and -1 were analyzed by means of a rotating-analyzer technique. To determine the parameters of polarization ellipse (the ellipticity angle $\varepsilon$ and the azimuthal angle $\theta$ of the major axis being counted counter-clockwise from the $\mathrm{X}$ axis), we used experimental dependences of the intensity I recorded by a photodetector on the analyzers' azimuth $\alpha$. They were fitted with a standard function

$$
I=I_{0} \frac{1+\cos 2 \varepsilon \cos [2(\alpha-\theta)]}{2} .
$$

Here $I_{0}$ denotes the intensity of the light incident at the analyzer. Examples of the experimental data and the fitting results are displayed in Fig. 1.

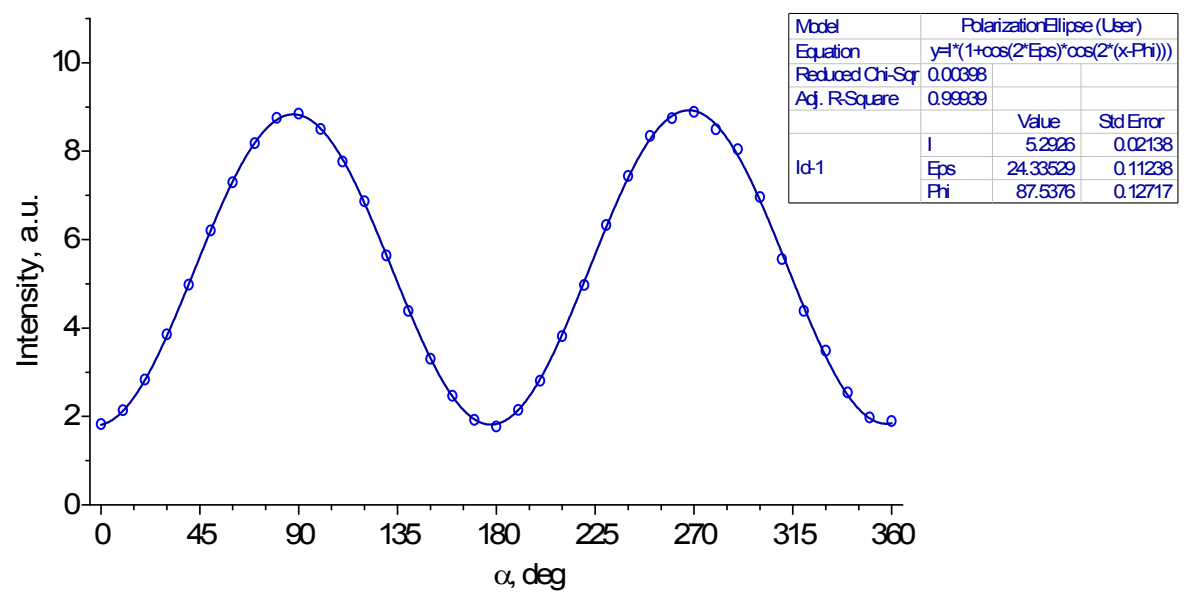

Fig. 1. Dependence of intensity of diffraction order transmitted through analyzer on analyzer's azimuth. Circles correspond to experimental data and smooth line to fitting.

Ukr. J. Phys. Opt. 2021, Volume 22, Issue 1 
The orientation and the ellipticity of the polarization ellipse were determined in the following way. Two kinds of the probing light waves were used in our experiments. First, we utilized a linearly polarized light. Four azimuthal positions of the input polarizer were chosen: 0, 45, 90 and $135 \mathrm{deg}$. The parameters of polarization ellipse of the light emergent from a sample (i.e., the azimuth $\theta$, the ellipticity angle $\varepsilon$ and the ellipticity itself, $e=\tan \varepsilon$ ) were determined experimentally for the following experimental conditions: (a) no ultrasound signal applied (i.e., a case of no diffraction occurring), (b) a zero-order optical beam under condition when an ultrasound signal is applied, and (c) the orders +1 and -1 of the diffracted optical beams under condition when an ultrasound signal is applied. The results obtained for the linearly polarized incident light are collected in Table 1.

Table 1. Polarization parameters of diffracted optical beams, as obtained for the linearly polarized input light.

\begin{tabular}{|c|c|c|c|c|}
\hline \multirow[b]{2}{*}{ № } & \multirow[b]{2}{*}{ Polarizer's azimuth $p, \operatorname{deg}$} & \multicolumn{3}{|c|}{ Output polarization } \\
\hline & & Azimuth $\theta$, deg & $\begin{array}{c}\text { Mean ellipticity } \\
\text { angle } \varepsilon, \text { deg }\end{array}$ & Ellipticity $e$ \\
\hline \multirow{5}{*}{1} & $p=0$ & & & \\
\hline & no diffraction & $-0.002 \pm 0.042$ & 0.136 & 0.0024 \\
\hline & zero order & $0.005 \pm 0.073$ & 1.334 & 0.0233 \\
\hline & -1 order & $2.096 \pm 0.557$ & 6.587 & 0.1155 \\
\hline & +1 order & $2.036 \pm 0.566$ & 7.033 & 0.1234 \\
\hline \multirow{5}{*}{2} & $p=90$ & & & \\
\hline & no diffraction & $90.062 \pm 0.028$ & 0.00036 & 0.0000062 \\
\hline & zero order & $90.225 \pm 0.148$ & 1.410 & 0.0246 \\
\hline & -1 order & $89.072 \pm 0.135$ & 2.832 & 0.0495 \\
\hline & +1 order & $89.146 \pm 0.074$ & 2.024 & 0.0353 \\
\hline \multirow{5}{*}{3} & $p=45$ & & & \\
\hline & no diffraction & $45.020 \pm 0.037$ & 0.000008 & 0.00000014 \\
\hline & zero order & $44.344 \pm 0.056$ & 0.925 & 0.0161 \\
\hline & -1 order & $65.145 \pm 0.175$ & 3.118 & 0.0545 \\
\hline & +1 order & $65.133 \pm 0.214$ & 2.461 & 0.0430 \\
\hline \multirow{5}{*}{4} & $p=135$ & & & \\
\hline & no diffraction & $135.008 \pm 0.078$ & 0.00013 & 0.0000023 \\
\hline & zero order & $136.042 \pm 0.258$ & 0.416 & 0.0073 \\
\hline & -1 order & $114.809 \pm 0.312$ & 3.336 & 0.0583 \\
\hline & +1 order & $115.023 \pm 0.272$ & 4.147 & 0.0725 \\
\hline
\end{tabular}

After that, we used a circularly polarized probing light. To provide the circular polarization, a quarter-wave plate was inserted behind the polarizer and in front of the sample. The fast axis of the quarter-wave plate was oriented at the azimuthal position $c=0^{\circ}$. The circularly polarized probing light waves of two alternative ('left' or 'right') handednesses were prepared via setting the polarizer into the azimuthal positions $p=45$ or $p=135 \mathrm{deg}$. The parameters $\theta, \varepsilon$ and $e$ of the polarization ellipse were determined experimentally under the same experimental conditions. The results obtained for the circularly polarized incident light are gathered in Table 2. 
Table 2. Polarization parameters of diffracted optical beams, as obtained for the circularly polarized input light.

\begin{tabular}{|c|c|c|c|c|}
\hline \multirow[b]{2}{*}{ № } & \multirow[b]{2}{*}{ Input polarization } & \multicolumn{3}{|c|}{ Output polarization } \\
\hline & & $\begin{array}{c}\text { Mean azimuth } \theta, \\
\text { deg }\end{array}$ & $\begin{array}{c}\text { Mean ellipticity angle } \\
\varepsilon, \text { deg }\end{array}$ & Ellipticity $e$ \\
\hline \multirow{5}{*}{1} & Left-handed circular & & & \\
\hline & no diffraction & - & 44.410 & 0.9796 \\
\hline & zero order & 17.679 & 43.862 & 0.9610 \\
\hline & -1 order & 87.538 & 24.335 & 0.4523 \\
\hline & +1 order & 87.638 & 24.479 & 0.4553 \\
\hline \multirow{5}{*}{2} & Right-handed circula & & & \\
\hline & no diffraction & - & 44.068 & 0.9680 \\
\hline & zero order & 161.540 & 43.589 & 0.9519 \\
\hline & -1 order & 89.459 & 24.343 & 0.4524 \\
\hline & +1 order & 89.449 & 24.504 & 0.4558 \\
\hline
\end{tabular}

\section{Discussion}

After analysis of the data presented in Table 1, one can assert that the linearly polarized light oriented 'horizontally' or 'vertically' hardly changes the polarization under AO interaction. This fact means that the above polarizations represent the eigenmodes for the experimental geometry under test. When the input linear polarization is set at the 'diagonal' angular positions (45 or $135 \mathrm{deg}$ ), the outgoing light polarization is rotated towards the 'vertical' $Y$ axis approximately by the angle $\Delta \theta \approx 20 \mathrm{deg}$ (see Fig. 2).

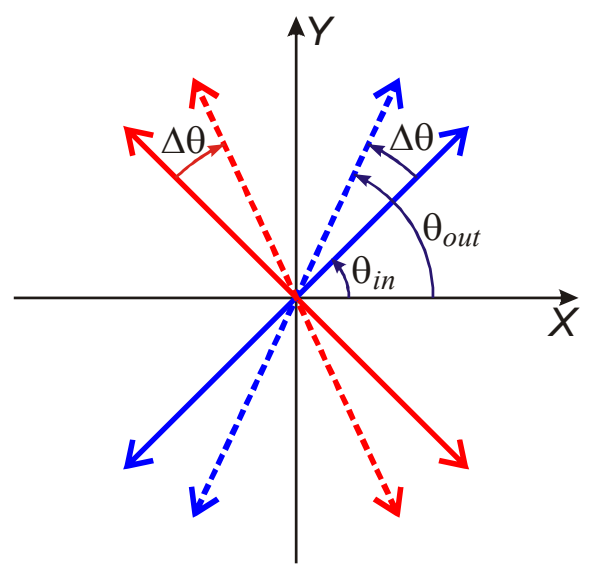

Fig. 2. Schematic representation of changes in the linear polarization azimuth occurring in the course of AO diffraction in fused silica. Solid lines correspond to input polarizations and dashed lines to polarizations of diffracted light. $\theta_{\text {in }}$ and $\theta_{\text {out }}$ denote the azimuths of input and output polarizations.

Let us remind the classification of AO interactions in isotropic solids suggested in Ref. [8]. When the incident light has the 'horizontal' polarization, we deal with the Type II of AO diffraction, which is governed by the EO coefficient $p_{11}$. In case of the 'vertical' polarization we arrive at the Type I of AO interactions, with the EO coefficient $p_{12}$ being of interest. Finally, when the incident light is linearly polarized at the azimuths 45 or $135 \mathrm{deg}$, both of the above types of AO diffraction occur simultaneously. Due to different diffraction efficiencies for the 'horizontally' and 'vertically' polarized optical modes, the amplitudes of the output-field components are proportional to the EO coefficients $p_{11}$ and $p_{12}$. Then it is evident that the azimuth $\theta_{\text {out }}$ of the resulting linear polarization is given by the relations 


$$
\begin{aligned}
& \tan \theta_{\text {out }}=p_{12} / p_{11}\left(\theta_{\text {in }}=45 \mathrm{deg}\right), \\
& \tan \theta_{\text {out }}=-p_{12} / p_{11}\left(\theta_{\text {in }}=135 \mathrm{deg}\right) .
\end{aligned}
$$

Following from the output polarization azimuths collected in Table 1, which are concerned with the input azimuths 45 and $135 \mathrm{deg}$, one can calculate the four $p_{12} / p_{11}$ ratios: $2.159 ; 2.158$; 2.163 and 2.142 , with the mean value amounting to $\left\langle p_{12} / p_{11}\right\rangle=2.155$. According to the handbook [9], the EO coefficients of fused silica are equal to $p_{11}=0.121$ and $p_{12}=0.270$. This gives the ratio $p_{12} / p_{11}=2.231$, which is very close to the mean value obtained from our experiments.

Now we proceed to the analysis of transformation of circularly polarized incident light at the AO diffraction in fused silica. The results of Table 2 lead to conclusion that the polarizations of the diffracted beams (the orders \pm 1 ) are elliptical, with the major axes of ellipses being oriented close to the $Y$ axis and the ellipticities $e$ being close to 0.45 . This is illustrated schematically in Fig. 3.

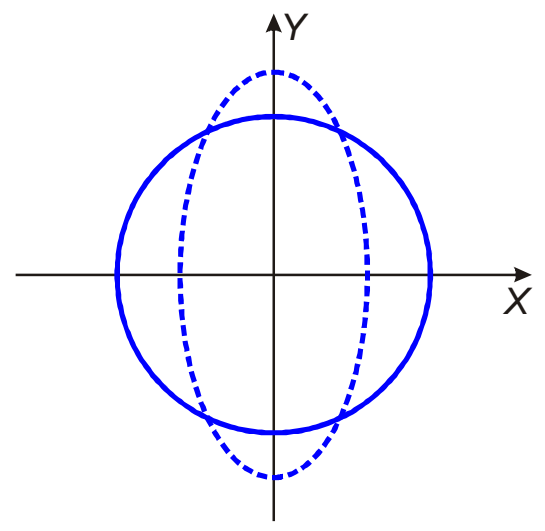

Fig. 3. Schematic representation of changes in the circular polarization occurring in the course of $A O$ diffraction in fused silica. Solid line corresponds to input polarization and dashed line to polarization of diffracted light.

The explanation of transformations of the circular polarization is very similar to that occurring in the case of linear polarization. The only difference is as follows: now the 'horizontal' and 'vertical' eigenmodes in the sample are shifted by the angle $\pm 90 \mathrm{deg}$. After AO interactions, the amplitudes of the output-field components differ due to different effective EO coefficients $p_{11}$ and $p_{12}$. A superposition of these orthogonal components gives the polarization ellipse, with the ellipticity $e$ equal to the ratio $p_{11} / p_{12}$ :

$$
e_{\text {out }}=\tan \left(\varepsilon_{\text {out }}\right)=p_{11} / p_{12} .
$$

Using the reference EO coefficients $p_{11}$ and $p_{12}$ specified above, one can calculate their ratio as $p_{11} / p_{12}=0.4481$. The ellipticities obtained experimentally (see Table 2 ) are equal to 0.4523 , $0.4553,0.4524$ and 0.4558 , whereas the mean value amounts to $\langle e\rangle=0.4539$. Therefore, the measurements of the polarization state for the light diffracted from the AO cell allow one to determine the ratio of EO coefficients $p_{11}$ and $p_{12}$ in the case of vitreous solids.

Another interesting fact can be extracted when analysing thoroughly the data of Table 1 . When the linearly polarized probing light is used, the diffracted beams become slightly elliptically polarized, with the ellipticity angle having the order of magnitude $\varepsilon \approx 3 \mathrm{deg}$. This could indicate that some phase retardation between the 'horizontal' and 'vertical' eigenmodes arises in the course of AO interaction, of which value can be estimated as $\varphi=2 \varepsilon \approx 6 \mathrm{deg}$. Clarification of this effect needs further studies based upon high-accuracy polarimetric techniques. 


\section{Conclusion}

In the present work we have studied the polarization of light diffracted at the longitudinal AW. The intermediate Bragg-Raman-Nath regime of diffraction in fused silica has been examined. The diffracted waves of the orders \pm 1 become elliptically polarized when the incident optical waves are linearly polarized with different azimuthal orientations or have left- or right-handed circular polarizations. For incident circularly (linearly) polarized waves the ellipticities (azimuths) of the diffracted waves are determined by the ratio of EO coefficients $p_{11}$ and $p_{12}$. The appropriate ratios determined from our polarization measurements are in good agreement with the data known from the open literature. Finally, a small deviation from the linear polarization state of the diffracted waves is detected when the linearly polarized light is incident on a sample. This deviation can be caused by changes in the phase difference of the diffracted eigenmodes.

\section{References}

1. Balakshyi V I, Paryhyn V N and Chyrkov L E. Physical principles of acousto-optics. Moscoe: Radio i Svyaz (1985).

2. Chang I C. Acousto-optic devices and application. In: Handbook of Optics. Ed. by Michael Bass, $2^{\text {nd }}$ Ed., Volume 2, McGraw-Hill, Inc. (1995).

3. Magdich L N and Molchanov V Ya, Acoustooptic devices and their application. Moscow: Sov. Radio (1978).

4. Kotov V M, Shkerdin G N and Shkerdin D G, 2002. Effect of acoustooptic Bragg diffraction on the degree of spatial coherence of a light beam. Opt. Spectrosc. 93: 291-294.

5. Chen-Wen Tarn, 1999. Spatial coherence property of a laser beam during acousto-optic diffraction. J. Opt. Soc. Amer. A. 16: 1395-1401.

6. Weng Cun-Cheng and Zhang Xiao-Man, 2015. Fluctuations of optical phase of diffracted light for Raman-Nath diffraction in acousto-optic effect. Chin. Phys. B. 24: 014210.

7. Martynyuk-Lototska I, Vasylkiv Y, Dudok T, Skab I and Vlokh R, 2018. Acoustooptic operation of optical vortex beams. Optik. 155: 179-184.

8. Mys O, Kostyrko M, Smyk M, Krupych O and Vlokh R, 2014. Anisotropy of acousto-optic figure of merit in optically isotropic media. Appl. Opt. 53: 4616-4627.

9. Pinnow D A. Elasto-optical materials. CRC Handbook of Lasers. Ed. by Pressley R J. Cleveland: The Chemical Rubber Co. (1971).

Krupych O., Martynyuk-Lototska I., Orykhivskyi I., Kostyrko M. and Vlokh R. 2021 Polarization of diffracted optical wave in the case of acousto-optic diffraction on the longitudinal acoustic wave in fused silica. Ukr.J.Phys.Opt. 22: 61 - 67. doi: 10.3116/16091833/22/1/61/2021

Анотація. Виконано поляриметричні дослідження дифрагованого світла, що з'являється внаслідок акустооптичної (АО) дифракції на поздовжній акустичній хвилі. Розглянуто випадок проміжного режиму дифракиії Брегга-Рамана-Ната в плавленому квариі. Показано, щзо еліптичність $і$ азимут дифрагованої хвилі визначається відношенням пружнооптичних коефіцієнтів $p_{11}$ і $p_{12}$. Визначене у такий спосіб відношення коефіиієнтів добре узгоджується з літературними даними. Виявлено незначне відхилення від лінійної поляризації для дифрагованих оптичних хвиль за умови, коли падаючі хвилі мають лінійну поляризацію. Це відхилення може бути викликане зміною різниці фаз дифрагованих власних хвиль.

Ukr. J. Phys. Opt. 2021, Volume 22, Issue 1 